Editorial

\title{
Acknowledgement to Reviewers of Advances in Environmental and Engineering Research in 2021
}

Advances in Environmental and Engineering Research Editorial Office

LIDSEN Publishing Inc., 2000 Auburn Drive, One Chagrin Highlands, Suite 200, Beachwood, OH, USA;

E-Mail: aeer@lidsen.com

Adv Environ Eng Res

2022, volume 3, issue 1

doi:10.21926/aeer.2201001
Received: January 06, 2022

Accepted: January 06, 2022

Published: January 06, 2022

\begin{abstract}
The editors of Advances in Environmental and Engineering Research would like to express their sincere gratitude to the following reviewers for assessing manuscripts in 2021. We greatly appreciate the contribution of expert reviewers, which is crucial to the journal's editorial process. We aim to recognize reviewer contributions through several mechanisms, of which the annual publication of reviewer names is one. Reviewers receive a voucher entitling them to a discount on their next LIDSEN publication and can download a certificate of recognition directly from our submission system. Additionally, reviewers can sign up to the service Publons (https://publons.com) to receive recognition. Of course, in these initiatives we are careful not to compromise reviewer confidentiality. Many reviewers see their work as a voluntary and often unseen part of their role as researchers. We are grateful to the time reviewers donate to our journals and the contribution they make.
\end{abstract}

If you are interested in becoming a reviewer for Advances in Environmental and Engineering Research, see the link at the bottom of the webpage http://www.lidsen.com/joinus.

The following reviewed for Advances in Environmental and Engineering Research in 2021:

\begin{tabular}{|l|l|l|}
\hline Adams, JamesB. & Hu, Zhengyi & Pytharoulis, Ioannis \\
\hline Albers, Sascha & Hua, Guanghui & Rashid, MuhammadImran \\
\hline Albini, Angelo & Hussein, Hussam & Ribeiro, FlaviaNoronhaDutra \\
\hline
\end{tabular}

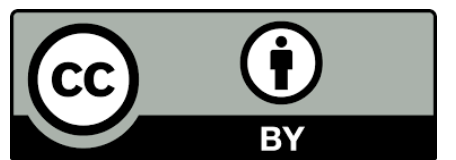

(c) 2022 by the author. This is an open access article distributed under the conditions of the Creative Commons by Attribution License, which permits unrestricted use, distribution, and reproduction in any medium or format, provided the original work is correctly cited. 


\begin{tabular}{|c|c|c|}
\hline Amponsah, William & Huthnance, JohnM. & Rigo, Tomeu \\
\hline Andersen, OleBaltazar & lacus, StefanoMaria & Saha, Asish \\
\hline Anderson, RichardH. & Iz, H.Bâki & Sarrà, Montserrat \\
\hline Aw, TiongGim & Jager, Henriette & Schüpbach, Beatrice \\
\hline Babaei, Hossein & Kabiri, Keivan & Selisteanu, Dan \\
\hline Bartolomeo, Nicola & Karagianni, Aikaterini & Shahzad, Muhammadımran \\
\hline Be'eri-Shlevin, Yaron & Kaskaoutis, Dimitris & Show, PauLoke \\
\hline Bełdowska, Magdalena & Khwarahm, NabazR. & Smaranda, Camelia \\
\hline Bełdowski, Jacek & Kowalczyk, Cezary & Smith, SiwapornMeejoo \\
\hline Bernardi, Bruno & Kurniawan, TonniAgustiono & Song, Zhanlong \\
\hline Besancenot, JeanPierre & Laca, Amanda & Srebotnjak, Tanja \\
\hline Bhaganagar, Kiran & Lepine, Christine & Steinemann, Anne \\
\hline Billock, VincentA. & Li, Ping & Strezov, Vladimir \\
\hline Chan, YiHerng & Li, Xinghua & Sun, Weiwei \\
\hline Chaturvedi, SudhirKumar & Lieske, DavidJ. & Tang, Mengling \\
\hline Chen, Chao & Liu, Cong & Teodoro, Ana \\
\hline Chen, Shaohua & Liu, Guorui & Thejll, PeterA. \\
\hline Chen, Yaning & Liu, Ruhai & Tiwari, ArjunPrasad \\
\hline Coccia, Mario & Liu, Wen-Cheng & vandoninck, Jasper \\
\hline Coman, Sonia & Lynch, ChristopherJ. & vanFraneker, Jan \\
\hline Cooke, RichardA. & Magazzino, Cosimo & Verichev, Konstantin \\
\hline Elsaid, Khaled & Mak, HugoWaiLeung & Vivoni, EnriqueR. \\
\hline Faiz, MuhammadAbrar & Marcano-Vega, Humfredo & Vrinceanu, NicoletaOlimpia \\
\hline Fang, Cheng & Michalet, Richard & Wang, Jinfeng \\
\hline Fernandez, Pilar & Mohamad, Diana & Wang, Shaoli \\
\hline Ganatsas, Petros & Molot, LewisA. & Wang, Zhifang \\
\hline Gao, Rui & Murty, P.L.N. & Wanner, Alice \\
\hline Generowicz, Agnieszka & Naz, BibiS. & Welker, Martin \\
\hline Goel, Gaurav & Nedjaï, Rachid & Yamano, Hiroya \\
\hline Gomes, Eduardo & Olie, Kees & Zamorska, Magdalena \\
\hline Gong, Guangcai & Omrany, Hossein & Zhang, Han \\
\hline Gophen, Moshe & Peng, Yutao & Zhang, Rong \\
\hline Guo, Bo & Potter, PhillipM. & Zhang, Wanchang \\
\hline He, Baojie & Povilaitis, Arvydas & Zhang, Xiu-Jun \\
\hline Hernández-Chover, Vicent & Hu, Zhengyi & Zhang, Xun \\
\hline
\end{tabular}


Adv Environ Eng Res 2022; 3(1), doi:10.21926/aeer.2201001

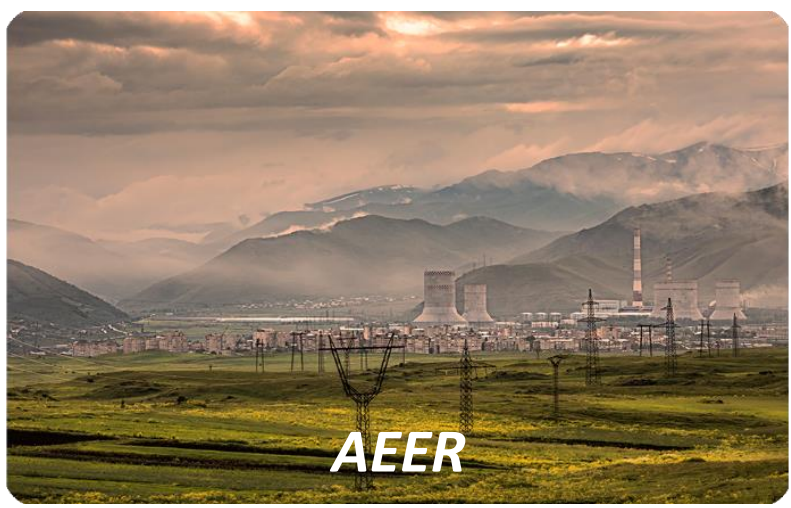

Enjoy $A E E R$ by:

1. Submitting a manuscript

2. Joining in volunteer reviewer bank

3. Joining Editorial Board

4. Guest editing a special issue

For more details, please visit:

http://www.lidsen.com/journals/aeer 